\title{
Dosimetric comparison of radiotherapy treatment plans done by IMRT and VMAT technique in head and neck cancer patients
}

\author{
Aparajeeta. ${ }^{1}$, Mehta A. ${ }^{2}$, Silambarasan N. ${ }^{3}$, Kumar P. ${ }^{4 *}$ \\ DOI: https://doi.org/10.17511/ijmrr.2021.i03.06 \\ 1 Aparajeeta, Junior Resident, Department of Radiation Oncology, Shri Ram Murti Institute of Medical Sciences, Bareilly, Uttar Pradesh, \\ India. \\ 2 Ankita Mehta, Senior Resident, Department of Radiation Oncology, Shri Ram Murti Institute of Medical Sciences, Bareilly, Uttar Pradesh, \\ India. \\ 3 N.S. Silambarasan, Assistant Professor cum Medical Physicist, Department of Radiation Oncology, Shri Ram Murti Institute of Medical \\ Sciences, Bareilly, Uttar Pradesh, India. \\ 4* Piyush Kumar, Professor and Head, Department of Radiation Oncology, Shri Ram Murti Institute of Medical Sciences, Bareilly, Uttar \\ Pradesh, India.
}

Background: The increasing patient load in radiotherapy centres demands selection of the technique that provides plans with optimal dosimetry in terms of target volume coverage, organs at risk (OAR) sparing and a lesser treatment time. This study was designed to compare the two widely practised conformal techniques, IMRT and VMAT in head and neck cancer patients in terms of planning target volume (PTV) coverage, OAR sparing and treatment delivery parameters. Materials and methods: For ten postoperative head and neck cancer patients who had been treated by IMRT technique virtual VMAT plans were generated for study purposes. The dose prescribed to PTV was 60 Gy in 30 fractions. The dose-volume parameters of PTV and OARs and the treatment delivery parameters were compared amongst both the techniques. Statistical significance was calculated using paired ' $t$ ' test. Results: Both the plans were comparable in terms of dosimetry. The only significant difference being better conformity in the IMRT plans. The dose to OARs was also comparable in both the techniques except for a significant reduction in the point dose to brainstem with the IMRT technique. Given the treatment delivery parameters, there was a significant reduction in the treatment delivery time and monitor units with the VMAT technique compared to the IMRT technique. Conclusion: VMAT technique gave comparable plans to that of the IMRT technique in terms of dosimetry but reduced the treatment time. It seems feasible in radiotherapy centres with increased patient load.

Keywords: 2019-nCoV, Wuhan, Coronavirus, Severe acute respiratory syndrome coronavirus, China

Corresponding Author

Piyush Kumar, Professor and Head, Department of Radiation Oncology, Shri Ram Murti Institute of Medical Sciences, Bareilly, Uttar Pradesh, India. Email: apaparajita@gmail.com

How to Cite this Article

To Browse

Aparajeeta, Mehta A, Silambarasan NS, Kumar P. Dosimetric comparison of radiotherapy treatment plans done by IMRT and VMAT technique in head and neck cancer patients. Int J Med Res Rev. 2021;09(03):167-172. Available From https://ijmrr.medresearch.in/index.php/ijmrr/article/ view/1295

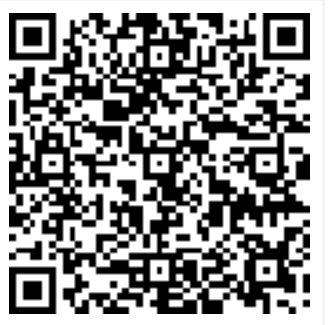

Manuscript Received 2021-05-16

Conflict of Interest No
Review Round 1 2021-05-26

Funding Nil
Review Round 2 2021-06-08

Ethical Approval Yes
Review Round 3 2021-06-18

Plagiarism X-checker $7 \%$
Accepted 2021-06-22

(c) 2021 by Aparajeeta, Ankita Mehta, N.S. Silambarasan, Piyush Kumar and Published by Siddharth Health Research and Social Welfare Society. This is an Open Access article licensed under a Creative Commons Attribution 4.0 International License https://creativecommons.org/licenses/by/4.0/ unported [CC BY 4.0]. 


\section{Background}

There has been an immense evolution in radiation planning of head or neck cancers. Because of the complex shape of the target volume and vicinity to a large number of organs at risk (OAR's) and minimal organ motion, the conformal radiotherapy planning techniques have offered significant dosimetric and clinical advantages. The treatment planning methods for head and neck cancers using external beam radiotherapy have evolved from the traditional three-field technique in the early days to intensity-modulated radiotherapy (IMRT), and recently to volumetric modulated arc therapy (VMAT). IMRT is an advanced form of threedimensional conformal radiotherapy (3D-CRT) that changes the intensity of radiation in different parts of a single radiation beam while the treatment is delivered. The inverse optimisation algorithm automatically determines the appropriate weights of segments to create a more uniform dose distribution throughout the target. [1]. VMAT includes a large number of beams from an arc projector with the dose being delivered dynamically with gantry rotation at each angle. [2]. The efficacy of IMRT over 3DCRT have been established in terms of better target volume coverage and organ at risk sparing and better clinical outcomes [3]. But the advantage of VMAT over IMRT remains controversial. This retrospective study was designed to compare these two techniques in post-operative head and neck cancer patients in terms of target volume coverage, organs at risk (OAR) sparing and treatment delivery time.

\section{Materials and Methods}

Study Setting: Department of Radiation Oncology, Shri Ram Murti Institute of Medical Sciences

Study Design: Retrospective study

Study population: Postoperative head and neck cancer patients treated between 2020-2021

Duration and type of study: A total of ten head and neck post-operative patients who had been treated by the IMRT technique were selected for the retrospective study. For each patient, a corresponding VMAT plan was generated for study purposes.

\section{Inclusion Criteria:}

01. Postoperative head and neck cancer patients

02. Conventional fractionation schedule-60 Gy/30
Fractions.

\section{Exclusion Criteria:}

01 . De novo patients with head and neck cancers

02. Patients with gross residual disease after surgery

\section{Radiotherapy planning}

All the patients underwent simulation in supine position with neck rest and shoulder traction using the 5-point thermoplastic cast. Contrast-enhanced CT scans of $3 \mathrm{~mm}$ slice thickness were obtained.

The following volumes were delineated:

Clinical Target Volume (CTV) primary- inclusive of post-operative bed and entire primary site Clinical Target Volume (CTV) nodal - nodal volumes were delineated as per the guidelines given by Biau $\mathrm{J}$ et al. [4]. Planning Target Volume (PTV)- $5 \mathrm{~mm}$ isotropic margin to the CTV to account for setup errors. The OAR'S were delineated as per DAHANCA guidelines. This included the Spinal cord, Mandible, Parotids, Lips, Left and Right Cochlea, Brainstem, Left and Right Eye, Left and Right Lens, Left and Right Optic nerve. An isotropic expansion of $5 \mathrm{~mm}$ for the PRV spine was given from the spinal cord and a $3 \mathrm{~mm}$ margin from the brainstem was given for the PRV brainstem.

\section{Dose prescription}

A total of $60 \mathrm{~Gy}$ in 30 fractions was prescribed to the PTV. All the patients received treatment using the IMRT technique. The constraints given for the OARs were:- PRV spine Dmax $\leq 50 \mathrm{~Gy}$; Mandible Dmax $\leq 70 \mathrm{~Gy}$; Lips $\leq 30 \mathrm{~Gy}$; PRV Brainstem Dmax 554Gy; Left and Right Cochlea Dmean $\leq 45 \mathrm{~Gy}$; Left and Right Parotid (combined) Dmean $\leq 26 \mathrm{~Gy}$; Left and Right Eye Dmax $\leq 50$ Gy; Left and Right Lens Dmax $\leq 7 \mathrm{~Gy}$; Left and Right Optic nerve Dmax $\leq 55 \mathrm{~Gy}$

\section{Planning}

1)The plans were created in Eclipse Treatment Planning System for Truebeam which is having the Millenium MLC.

2)Inverse planning with one or multiple optimizations and running was done to achieve the target dose distribution and OAR sparing. AAA algorithm was used for dose calculation after the optimization process.

3)The OAR dose constraints and target dose parameters were followed as per RTOG guidelines and evaluated as per ICRU 83 recommendations. 
4)In IMRT, 9 fields were used such as $0^{\circ}, 40^{\circ}, 80^{\circ}, 120^{\circ}, 160^{\circ}, 200^{\circ}, 240^{\circ}, 280^{\circ}, 320^{\circ}$ to achieve the goals whereas in VMAT plan 2 full arcs were used (ARC-I $181.1^{\circ}$ to $179.9^{\circ}$ and ARC-II $179.9^{\circ}$ to $181.1^{\circ}$ clockwise and counter-clockwise arcs respectively.

\section{Data collection}

The following dosimetric parameters were assessed and compared amongst the two planning techniques:

01. PTV- D95\%, D50\%, D2\% (Dnear max), D98\% (Dnear min), Conformity Index (CI) (ICRU 62) [5], Homogeneity Index (HI) (ICRU 83) [6].

02. Organs at risk (OARs)

Dmax was calculated for serial organs (spinal cord, brainstem, mandible, eyes, lens, optic nerve, optic chiasma) and Dmean was calculated for parallel organs (parotid, cochlea, lips).

03. Treatment delivery parameters- Treatment delivery time, Monitor Units (MUs)

Statistical analysis:Statistical significance was calculated using paired ' $t$ ' test. A $p$-value of $<0.05$ was considered as statistically significant.

\section{Results}

The dosimetry of PTV showed comparable coverage in both the techniques in terms of $D 95 \%(p=0.773)$, $\mathrm{D} 50 \%(p=0.093)$. The near Dmax (D2) $(p=0.126)$ and near Dmin (D98) $(p=0.569)$ were also similar with no significant difference in both the techniques and fulfilled the pre specified objective. The prespecified objective was fulfilled with both the techniques with more than $95 \%$ of the volume receiving $95 \%$ of the prescribed dose. The dose homogeneity was also similar but conformity was significantly better in the IMRT technique $(\mathbf{p}<\mathbf{0 . 0 0 2})$ (Table 1 )

Table-1: Dosimetric parameters of the PTV in two techniques (Mean \pm SD)

\begin{tabular}{|l|l|l|l|}
\hline \multicolumn{1}{|c|}{ Parameters } & \multicolumn{1}{c|}{ IMRT } & \multicolumn{1}{c|}{ VMAT } & p-value \\
\hline D95\% & $58.59 \pm 0.68$ & $58.68 \pm 0.66$ & 0.773 \\
\hline D50\% & $60.5 \pm 0.44$ & $60.69 \pm 0.34$ & 0.093 \\
\hline D2\% (Dnear max) & $61.71 \pm 0.68$ & $62.02 \pm 0.27$ & 0.126 \\
\hline D98\% (Dnear min) & $57.71 \pm 0.85$ & $57.47 \pm 0.99$ & 0.569 \\
\hline Conformity Index (CI) & $1.14 \pm 0.09$ & $1.29 \pm 0.13$ & 0.002 \\
\hline Homogeneity Index (HI) & $0.06 \pm 0.01$ & $0.07 \pm 0.01$ & 0.147 \\
\hline
\end{tabular}

Table-2: Dosimetric parameters of the OARs in two techniques (Mean \pm SD)

\begin{tabular}{l|l|l|l} 
OARs & IMRT & VMAT & p-value \\
\hline
\end{tabular}

\begin{tabular}{|l|l|l|l|}
\hline Brainstem & $35.59 \pm 5.82$ & $39.05 \pm 4.32$ & 0.003 \\
\hline Parotid & $34.28 \pm 7.86$ & $37.15 \pm 6.43$ & 0.052 \\
\hline Left cochlea & $20.32 \pm 12.04$ & $20.3 \pm 9.36$ & 0.994 \\
\hline Right cochlea & $12.19 \pm 5.53$ & $13.49 \pm 5.87$ & 0.652 \\
\hline Lips & $31.56 \pm 5.77$ & $29.19 \pm 1.61$ & 0.155 \\
\hline PRV spine & $39.54 \pm 2.65$ & $40.18 \pm 2.35$ & 0.525 \\
\hline Mandible & $62.48 \pm 1.05$ & $63.1 \pm 0.49$ & 0.076 \\
\hline
\end{tabular}

In terms of dosimetry of OARs, there was a significant reduction in the maximum dose of the brainstem with the IMRT technique compared to the VMAT technique but the absolute doses were far lesser than the prespecified constraint for the brainstem (Dmax $=54 \mathrm{~Gy}$ ). In terms of dosimetry of other OARs, a significant difference could not be found amongst both the techniques (Table 2).

Table-3: Treatment delivery parameters of the two techniques

\begin{tabular}{|l|l|c|c|}
\hline \multicolumn{1}{|c|}{ Parameters } & \multicolumn{1}{c|}{ IMRT } & VMAT & p-value \\
\hline $\begin{array}{l}\text { Treatment delivery time (in } \\
\text { minutes) }\end{array}$ & $3.95 \pm 0.85$ & $1.21 \pm 0.34$ & $<0.0001$ \\
\hline Monitor Units (MUs) & $1581.6 \pm 341.78$ & $486.9 \pm 136.23$ & $<0.0001$ \\
\hline
\end{tabular}

VMAT plans showed a significantly reduced treatment delivery time as well as a reduced number of monitor units over IMRT plans. (Table 3 )

\section{Discussion}

In our study, the dose distribution of the target volume and OAR sparing was comparable amongst both the techniques. Although statistically significant improvement in conformity was seen with the IMRT technique, this did not translate into better sparing of OARs with IMRT. The reduction in the maximum dose to the brainstem in the IMRT technique was possibly due to better conformity of the IMRT plans. A marked difference was a reduction in treatment delivery time (3.95 \pm 0.85 mins for IMRT vs $1.21 \pm 0.34$ mins for VMAT; $p$ $<0.0001)$. This was reduced to nearly one third in the VMAT technique.

A similar trend was observed in Monitor Units showing a significant reduction in VMAT technique $(1581.6 \pm 341.78$ vs $486.9 \pm 136.23, p<0.0001)$. As per the findings of our study, VMAT largely reduces the treatment delivery times but did not compromise the planning quality. The reduction in monitor units with the VMAT technique will possibly reduce the total body exposure and the integral dose. Because of the increasing patient load in the radiotherapy department over the period, the waiting time and smooth running become cumbersome. 
The implementation of the VMAT technique offers an efficient solution to this problem with optimal radiotherapy plans. This is particularly because of the reduction in treatment delivery time by VMAT technique as fewer MUs are required to deliver the prescribed dose with arc beam arrangement. Also chances of intra fraction motion are minimized. The treatment time saved can be utilized in the implementation of more online imaging technologies. But the associated problem is that the VMAT technique is known to have a learning curve and requires a much higher planning time. Studenski M T et al in their study on 20 patients found no significant difference in the dosimetric parameters for both IMRT and VMAT techniques. But there was a significant reduction in treatment delivery times with VMAT plans with an average reduction of $9.2 \pm 3.9$ min over IMRT plans.

Also, there was a significant reduction in monitor units. [7]. In our study also there was a reduction in treatment delivery times by $2.74 \pm 0.51$ mins in VMAT plans over IMRT plans. This study validated the findings of our study with comparable dosimetry amongst both IMRT and VMAT plans but a significant reduction in the treatment delivery time and MU's. Holt $A$ et al in a multi-institutional study showed better sparing for almost all OARs with VMAT. There was no significant difference in the PTV coverage, dose homogeneity and conformity. There was a significant reduction in the dose to parotid glands and oral cavity with VMAT technique compared to IMRT technique (27.2Gy vs 25Gy for parotids and $39.4 \mathrm{~Gy}$ vs $36.7 \mathrm{~Gy}$ for oral cavity, $\mathrm{p}$ $<0.001)$.

This was contrary to our findings the possible reason being that in their study the planning time and methodology of the VMAT technique was more sophisticated. Similar to our study this study also showed a significant reduction in treatment delivery times with VMAT plans (13.15 min for IMRT vs 5.54 min for VMAT). [8]. Kryger $M$ et al in a study on 14 patients comparing the dosimetric parameters showed that the VMAT and IMRT plans were comparable in conformity and homogeneity. However, OAR sparing was better seen with few of the IMRT plans over VMAT plans in terms of a significant reduction in the dose to oral cavity and constrictors while the VMAT technique reduced the maximum dose delivered to the spinal cord (1800cGy lower than IMRT plans, $\mathrm{p}<0.001$ ). There was a significant reduction in the treatment delivery time and MUs with VMAT plans. [9].
Although few studies have shown better planning with VMAT over IMRT technique while others have shown comparable dosimetric parameters with both techniques. [10-14] With a growing incidence of cancer, there is an increase in the patient burden over centres and to have a smooth and efficient running we need to optimize the treatment delivery as well as planning times. Therefore, a validation of the contemporary conformal technique, IMRT and VMAT in terms of dosimetric comparison, and treatment time are needed. In our study, conformity was significantly inferior in VMAT plans even though the results will not have a clinical impact or an impact on the dosimetric parameters of the OAR's. The treatment planning time will play a significant role in VMAT for one accepted plan will take approximately 4-5 hours whereas in IMRT, it will take only half an hour. A few VMAT plans are having lesser conformity as serial OARs are located close to the target and we need to consider saving the OAR thus losing the target dose distribution. In case we would have used the high definition MLCs, because of the lesser thickness of the MLC, it might play a major role in sparing the OARs. Possibilities of improving the target dose distribution are also there. Our study has two limitations. Firstly, the sample size is small and secondly, long term clinical outcomes are not known. Most of the studies in the literature have compared dosimetry only and an emphasis on the clinical outcomes and toxicity has not been laid. Large scale studies with clinical outcomes are required to support the widespread implementation of the VMAT technique.

\section{Conclusion}

The study highlights that the VMAT technique can be safely implemented in post-operative patients with head and neck cancers and will reduce the delivery times compared to the IMRT technique. This technique may be useful for radiotherapy centres with increasing patient load to decrease the waiting list.

\section{What does the study add to the existing knowledge?}

The present study demonstrated that the newer radiotherapy VMAT technique is comparable to the IMRT technique in terms of dosimetric parameters and can be safely utilized for treating post-operative cancer patients of head and neck with the added advantage of lesser treatment time. 


\section{Author's Contribution}

Dr Aparajeeta: Statistical analysis, Drafting and editing the manuscript

Dr. Ankita Mehta: Collected data, Verification of data, Drafting the manuscript

Mr. Silambarasan NS: Treatment planning, Drafting the manuscript

Dr. Piyush Kumar: Study designing, Manuscript editing, Finalising and intellectual content

\section{Reference}

01. Taylor A, Powell ME. Intensity-modulated radiotherapy-what is it?. Cancer Imaging. $2004 \mathrm{Mar}$ 26;4(2)68-73. doi: 10.1102/1470-7330.2004.0003 [Crossref][PubMed][Google Scholar]

02. Vanetti E, Clivio A, Nicolini G, Fogliata A, GhoshLaskar S, Agarwal JP, et al. Volumetric modulated arc radiotherapy for carcinomas of the oro-pharynx, hypo-pharynx and larynx- a treatment planning comparison with fixed field IMRT. Radiother Oncol. 2009;92(1)111-7. doi: 10.1016/j.radonc. 2008.12.008 [Crossref][PubMed][Google Scholar]

03. Teoh M, Clark CH, Wood K, Whitaker S, Nisbet A. Volumetric modulated arc therapy- a review of current literature and clinical use in practice. $\mathrm{Br} \mathrm{J}$ Radiol. 2011;84(1007)967-96. doi: 10.1259/bjr /22373346 [Crossref][PubMed][Google Scholar]

04. Biau J, Lapeyre M, Troussier I, Budach W, Giralt J, Grau C, et al. Selection of lymph node target volumes for definitive head and neck radiation therapy- a 2019 Update. Radiother Oncol. 2019 May;134;1-9. doi: 10.1016/j.radonc.2019.01.018 [Crossref][PubMed][Google Scholar]

05. Stroom JC, Heijmen BJ. Geometrical uncertainties, radiotherapy planning margins, and the ICRU-62 report. Radiother Oncol. 2002 Jul;64(1)75-83. doi: 10.1016/s01678140(02)00140-8 [Crossref][PubMed][Google Scholar]

06. Hodapp N. Der ICRU-Report 83- Verordnung, Dokumentation und Kommunikation der fluenzmodulierten Photonenstrahlentherapie (IMRT) [The ICRU Report 83- prescribing, recording and reporting photon-beam intensity-modulated radiation therapy (IMRT)]. Strahlenther Onkol. 2012 Jan;188(1)97-9. German. doi: 10.1007/s00066011-0015-x [Crossref][PubMed][Google Scholar]
07. Studenski MT, Bar-Ad V, Siglin J, Cognetti D, Curry J, Tuluc $M$, et al. Clinical experience transiti oning from IMRT to VMAT for head and neck cancer. Med Dosim. 2013;38(2)171-5. doi: 10.1016/j.medd os.2012.10.009[Crossref][PubMed][Google Scholar]

08. Holt A, Van Gestel D, Arends MP, Korevaar EW, Schuring D, Kunze-Busch MC, et al. Multiinstitutional comparison of volumetric modulated arc therapy vs intensity-modulated radiation therapy for head-and-neck cancer- a planning study. Radiat Oncol. 2013 Jan 31;8;26. doi: 10.1186/1748-717X8-26 [Crossref][PubMed][Google Scholar]

09. Kryger M, Wang W, Wu S P P, Xue J, et al. A Comparison of IMRT and VMAT Treatment Planning for Head and Neck Cancer With NTCP/TCP Analysis. Int J Radia Oncol, Bio, Phys. 2017;99(2)E680. [Crossref][PubMed][Google Scholar]

10. Johnston M, Clifford S, Bromley R, Back M, Oliver $L$, Eade $T$. Volumetric-modulated arc therapy in head and neck radiotherapy- a planning com parison using simultaneous integrated boost for nasopharynx and oropharynx carcinoma. Clin Oncol R Coll Radiol. 2011;23(8)503-11. doi: 10.1016/j.clo n.2011.02.002[Crossref][PubMed][Google Scholar]

11. Wiezorek T, Brachwitz T, Georg D, Blank E, Fotina I, Habl G, et al. Rotational IMRT techniques compared to fixed gantry IMRT and tomotherapymulti-institutional planning study for head-and-neck cases. Radiat Oncol. 2011;6;20. doi: 10.1186/1748717X-6-20 [Crossref][PubMed][Google Scholar]

12. Rao M, Yang W, Chen F, Sheng K, Ye J, Mehta V, et al. Comparison of Elekta VMAT with helical tomotherapy and fixed field IMRT- plan quality, delivery efficiency and accuracy. Med Phys. 2010 Mar;37(3)1350-9. doi: 10.1118/1.3326965 [Crossref][PubMed][Google Scholar]

13. Verbakel WF, Cuijpers JP, Hoffmans D, Bieker M, Slotman BJ, Senan S. Volumetric intensitymodulated arc therapy vs conventional IMRT in head-and-neck cancer- a comparative planning and dosimetric study. Int J Radiat Oncol Biol Phys. 2009;74(1)252-9. doi: 10.1016/j.ijrobp.2008.12. 033 [Crossref][PubMed][Google Scholar]

14. Cozzi L, Fogliata A, Bolsi A, Nicolini G, Bernier J. Three-dimensional conformal vs intensity-modulated radiotherapy in head-and-neck cancer patientscomparative analysis of dosimetric and technical parameters. Int J Radiat Oncol Biol Phys. 2004; 58(2)617-24. doi: 10.1016/j.ijrobp.2003.09.059 [Crossref][PubMed][Google Scholar] 